\title{
Accurate Free Vibration Analysis of Launcher Structures Using Refined 1D Models
}

\author{
Erasmo Carrera*, Enrico Zappino** and Tommaso Cavallo*** \\ Department of Mechanical and Aerospace Engineering, Politecnico di Torino, Corso Duca degli Abruzzi 24, Torino 10129, Italy
}

\begin{abstract}
This work uses different finite element approaches to the free vibration analysis of reinforced shell structures, and a simplified model of a typical launcher with two boosters is used as an example. The results obtained using a refined one-dimensional (1D) beam model are compared to those obtained with commercial finite element software. The 1D models that are used in the present work are based on the Carrera Unified Formulation (CUF), which assumes a variable kinematic displacement field over the cross-sections of the beam. Two different sets of polynomials that correspond to Taylor (TE) or Lagrange (LE) expansions were used. The analyses focused on three reinforced structures: a stiffened panel, a reinforced cylinder and the complete structure of the launcher. The frequencies and natural modes obtained using one-dimensional models are compared to those obtained from classical finite element analysis. The classical FE models were built using a beam-shell or solid elements, and the results indicate that the refined beam models can in fact be used to investigate the behavior of very complex reinforced structures. These models can predict the shell-like modes that are typical of thin-walled structures that cannot be detected using classical beam models. The refined 1D models used in the present work provide results that are as accurate as those from solid FE models, but the 1D models have a much lower computational cost.
\end{abstract}

Key words: Launchers Analysis, CUF, Structural Dynamics, Refined Beam Models

\section{Introduction}

Launchers have been and will be essential for past and future space exploration as well as for putting satellites in orbit to provide communications and to observe the Earth. Launchers are made using reinforced thin-walled structures that are very complex and must survive under severe loading conditions. Therefore, it is necessary to develop advanced numerical tools to reduce the computational cost of the complex experimental activities that are required during structural design.

Computational tools have been used since the sixties to predict the dynamic characteristics of rockets and launchers. Wempner and Wilms [1] developed a rigid body model with six degrees of freedom (DOF) to model the dynamic characteristics of multi-staged, free-flight, ballistic rockets. Unfortunately, the capabilities of computers of that time were not sufficient to conduct an analysis of very complex structures. Modal synthesis or component mode techniques were thus widely used to conduct a dynamic analysis of large structures by dividing these into substructures or components. In 1963, Przemieniecki [2] introduced a matrix approach to evaluate the stresses and deflections in an aircraft structure composed of a number of substructural components. Each sub-structure was first analysed separately, and then equilibrium equations at the boundaries were used to ensure the congruence of the different substructures. In 1965, Hurty [3] conducted a dynamic analysis of complex structural systems by using a component modes approach. The generalized matrices for the mass, stiffness and damping were determined for each component, and the congruence equations were used at the interface to fulfil the requirements for continuity. At that time, Craig Bampton [4] proposed a method to study a complex structure as an
This is an Open Access article distributed under the terms of the Creative Commons Attribution Non-Commercial License (http://creativecommons.org/licenses/by$\mathrm{nc} / 3.0 /$ which permits unrestricted non-commercial use, distribution, and reproduction in any medium, provided the original work is properly cited. (c) $\quad$ * Professor of Aerospace Structures and Aeroelasticity.

** Research Assistant, Ph.D. , Corresponding Author, e-mail: enrico.zappino@polito.it.

*** Ph.D Student. 
assemblage of various sub-structures by considering basic mass and stiffness matrices. The conditions for geometrical compatibility were used along the boundaries of the substructures, and the Rayleigh-Ritz procedure was used to reduce the total number of DOFs. In 1970, Grimes et al. [5] performed an experimental test campaign on a 1/10 scale model to evaluate the dynamic behaviour of the Saturn V launcher and compared the results obtained with those obtained using theoretical models. In 1971, Benfield and Hruda [6] presented a method to determine the natural modes of a complex structure by means of the component vibration modes. In this case the modes for each substructure were separately determined and were then used to synthesize the global model. The number of modes that were introduced for each component can be truncated, and in this way, the number of generalized coordinates that are required for a dynamic analysis can be reduced. Goldenberg and Shapiro [7] formulated and evaluated various approaches that coupled two or more simple substructures in order to investigate complex systems using data obtained by analysing individual components. In 1976, Agrawal [8] used a model synthesis technique to investigate the normal modes, natural frequencies and dynamic responses of a three-dimensional complex structure with flexible joints. The equations of motion were developed by means of Lagrange's equations. Thornton [9] determined the natural frequencies and the modal shapes of a 1/15 scaled Space Shuttle using a beam model by representing the stiffness and mass properties of the physical structure. In 1989, Urgueira [10] analysed a sub-structures coupling technique that incorporates the data available from the modal experimental test. Two coupling approaches were investigated: impedance and modal coupling techniques. The two techniques were compared to determine the main sources of errors in predicting the dynamic behavior that is to be identified.

Nowadays, very fast computers and clusters are available, and these enable the analysis of highly complex structures. However, the methods mentioned above are still used during the preliminary design of a structure. Finite Element Models (FEM) can be used to analyze very complex structures, including mechanical parts, engines, aircraft frames, and complete launcher structures. In 1996, Friswell and Mottershead [11] presented a book on the finite element model where data acquired from an experimental vibration test was used to update the numerical model. Buehrle et al. [12] used an FE model to conduct the static analysis of an aircraft fuselage by considering beams, plates and solid elements within the same model. In 2009, $\mathrm{Hu}$ et al. [13] conducted a structural dynamics analysis of a sounding rocket during the lift-off phase, and the nose fairing, payload, ground support frame and boosters were included in the full finite element model. De Vivo et al. [14] used an alternative method to carry out the dynamic analysis of complex structures in 2010 by using the natural excitation technique in order to achieve the modal parameters from the wind force for the Vega launcher, which is the new European launcher. In 2013, Fransens et al. [15] adopted operational modal analysis tools to verify the performance of a solid rocket booster of the Vega Launcher. One year later, Işik et al. [16] published an article on using a finite element model to model of complete launcher system by considering both the normal vibration modes and the constraint modes.

The complete schema of a launcher, see Fig. 1, can be discretized using classical elements where both longitudinal and transversal stiffeners and skins are used to both withstand the high levels of stress and to reduce weight. Moreover, this configuration allows for concentrated loads to be applied and openings to be introduced into the structure. These structures are highly complex and require the use of Finite Element Methods (FEM) where solid (3D), shell (2D) and beam (1D) elements can be used to describe the kinematics of the components. Another issue that is important when modelling reinforced structures is to properly represent the configuration of the structure according to the technological process that is used to build it. The stiffness of the interface between the skin and the reinforcements can be affected by the coupling approach that is used between the two components. Two different methods are generally used, one involves the use of rivets or welding and the latter uses Computer Numerical Control (CNC) techniques. The two different joints are shown in Fig. 2. When a CNC approach is used, such as milling (Fig. 2a), continuity is guaranteed between the panel and the stringer. On the other hand, as is shown in Fig. 2b, classical joints can be used as welding and rivets, and the congruence of the displacements can be verified only in part of the interface.

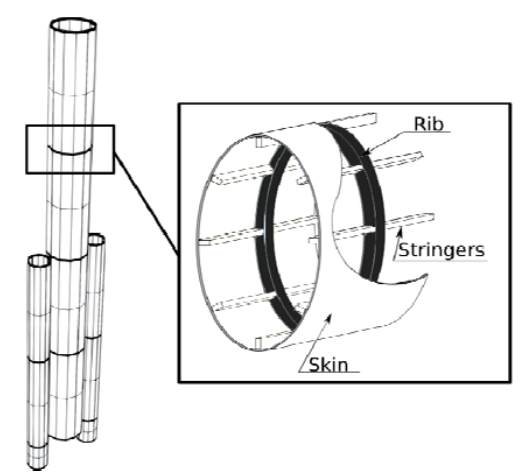

Fig. 1. Example of a thin-walled reinforced structure: a launcher structure 
Moreover, the joints may have a lower strength than that of the original material. When stringers and skin are a unique component, only solid elements are representative of the structure (see Fig. 3a), and the results can be very accurate. If the structure is welded, shell and beams elements can be used to simulate the mechanical behaviour (see Fig. 3b and c), introducing acceptable errors in both the discretization and the results. However, 2D-1D elements are normally used because 3D elements introduce a high number of DOFs. In this sense, the refined 1D models that are introduced in this work are very attractive because they ensure the structural continuity between the skin and the stringers as a solid model and provide a quasi three-dimensional solution with a lower number of DOFs. In fact, the geometrical aspect-ratio should not exceed the critical value when solid elements are used. Therefore, when thin-walled structures are considered, a higher number of elements is required as a result of the small dimension of the thickness. The use of refined one-dimensional models splits the solution into two components, one along the beam axis and one over the cross-section. Therefore, there are no limitations to the value of the aspect-ratio. When 1D-2D FE models are used, the introduction of stringers requires the use of classical beam elements that do not provide accurate local results.

There are a number of efficient computational methods that can be used to analyse reinforced shell structures, and

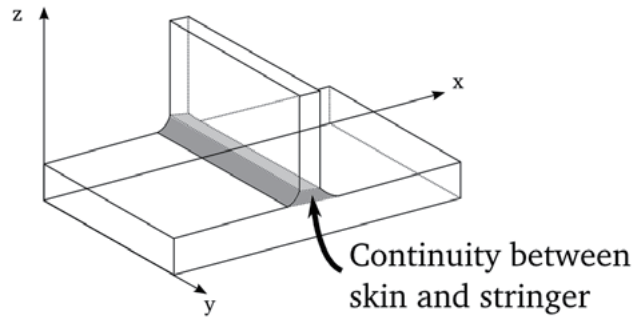

(a) Milled this work considers the one-dimensional variable kinematic finite element formulation based on the Carrera Unified Formulation (CUF). The CUF was initially developed for plate/shell models $[17,18,19]$ and was later extended to onedimensional models [20]. When CUF is applied to a beam model, the displacement field is obtained as the product of two function expansions: one defined over the crosssection and one along the beam axis. The Taylor expansion (TE) or Lagrange expansion (LE) can be used to describe the displacement over the cross-section. When TE is adopted, as shown by Carrera and Giunta [20] and Carrera et al. [21], the displacements are approximated using a global expansion around a point, which is usually the centroid of the crosssection. Static and dynamic analyses using TE models were presented in Refs. [22] and [23]. When LE is adopted [24, $25]$, the cross-section is discretized using the 2D Lagrange elements, and the displacements are expressed as a function of the displacements in the cross-sectional nodes, which are the only unknowns. The CUF allows the TE and LE models to be obtained without the need for ad hoc formulations. When TE is used, the order of the expansion $\mathrm{N}$ can be changed as a free parameter of the analysis. Classical models, such as Euler-Bernoulli and Timoshenko, can be obtained as a particular case of the TE formulation. When an LE expansion is considered, the solution can be improved using a quadratic element instead of a linear element or refining the mesh over

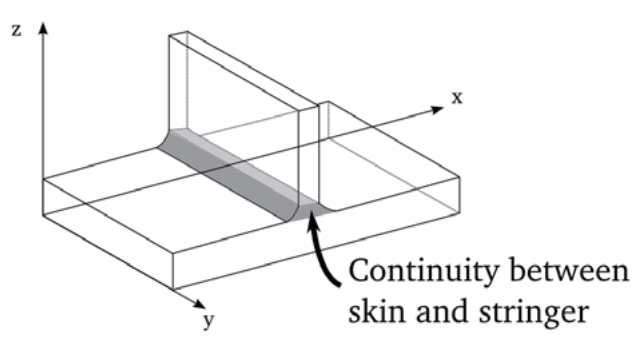

(b) Welded

Fig. 2. Comparisons of different connection between panel and reinforcement

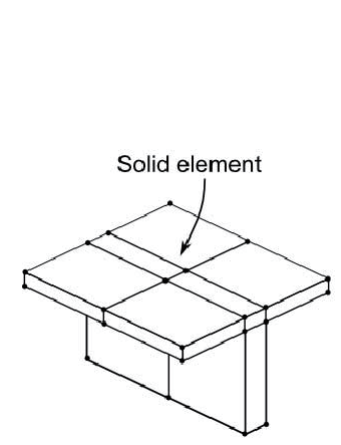

(a) Solid model

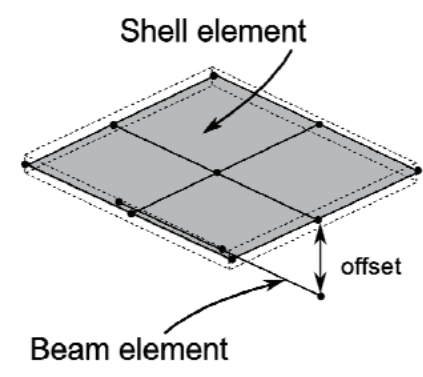

(b) Shell-Beam model

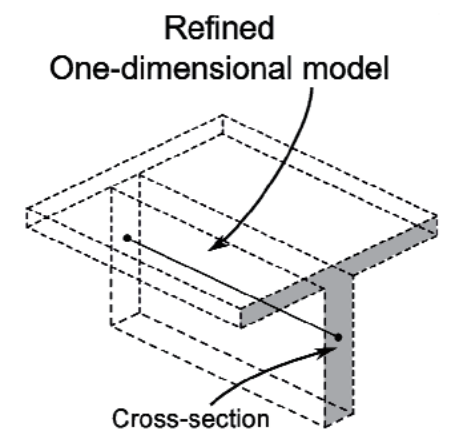

(c) 1D Refinded model

Fig. 3. Comparison between different approaches in the analysis of reinforced structures 
the cross-section. In both formulations, the finite element method is used to solve the problem along the axis in order to handle arbitrary geometries, boundary and loading conditions. Refined one-dimensional models provide a quasi-3D solution, and the present model can provide local modal shapes on both skin and stringers. On the other hand, no accurate information is available for the stringers when 2D-1D models are used. An example of these capabilities in shown in Refs. [26, 27], which present a component-wise (CW) approach that will be used in this paper. The higherorder beam models used in the present work were applied to the dynamic analysis of a stiffened plate, a reinforced cylinder and the complete structure of the launcher. The stringers were placed along both the longitudinal and transversal directions, and the natural frequencies and modal shapes were used to verify the accuracy of the model. Particular attention was given to a comparison between the

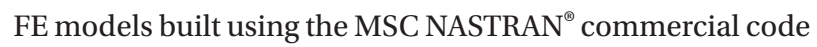
with both Solid and Shell/Beam elements.

\section{Refined one-dimensional models}

This section introduces a unified formulation for refined one-dimensional models, and for the sake of brevity only main features are reported in this work. More details can be found in Ref. [27].

\subsection{Preliminary}

The reference frame that was adopted is shown in Fig.4. The y-axis is the beam axis and the beam length is $L$. The displacement vector $u(x, y, z)$ can be expressed through the $\mathrm{u}_{\mathrm{x}}, \mathrm{u}_{\mathrm{y}}$ and $\mathrm{u}_{\mathrm{z}}$ components as:

$$
u(x, y, z)=\left\{\begin{array}{lll}
u_{x} & u_{y} & u_{z}
\end{array}\right\}^{T}
$$

The superscript $\mathrm{T}$ denotes transposition. Similarly, the

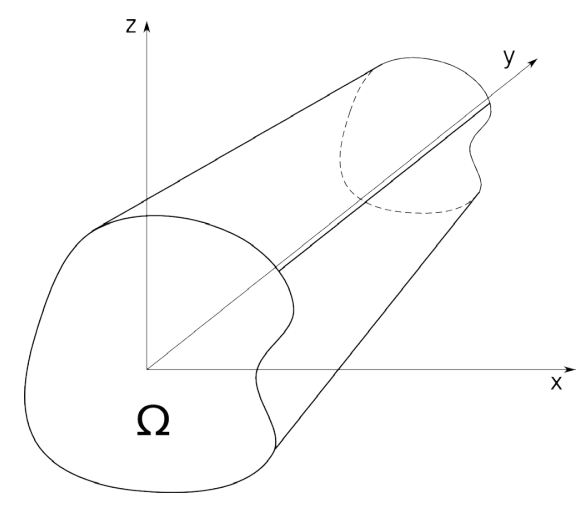

Fig. 4. One-dimensional model reference system. stress $\sigma$ and the strain $\varepsilon$ can be introduced as follows:

$$
\begin{aligned}
& \sigma=\left\{\begin{array}{llllll}
\sigma_{x x} & \sigma_{y y} & \sigma_{z z} & \sigma_{x y} & \sigma_{x z} & \sigma_{y z}
\end{array}\right\}^{T} \\
& \varepsilon=\left\{\begin{array}{lllllll}
\varepsilon_{x x} & \varepsilon_{y y} & \varepsilon_{z z} & \varepsilon_{x y} & \varepsilon_{x z} & \varepsilon_{y z}
\end{array}\right\}^{T}
\end{aligned}
$$

Linear strain-displacement relations are used:

$$
\varepsilon=D u=\left(\left\lfloor D_{y}\right\rfloor+\left[D_{\Omega}\right]\right) u
$$

$\mathrm{D}$ is a differential operator, and the explicit formulation can be found in the CUF book [28].

$$
D=\left[\begin{array}{ccc}
\frac{\partial}{\partial x} & 0 & 0 \\
0 & \frac{\partial}{\partial y} & 0 \\
0 & 0 & \frac{\partial}{\partial z} \\
\frac{\partial}{\partial y} & \frac{\partial}{\partial x} & 0 \\
\frac{\partial}{\partial z} & 0 & \frac{\partial}{\partial x} \\
0 & \frac{\partial}{\partial z} & \frac{\partial}{\partial y}
\end{array}\right]=\left[\begin{array}{ccc}
\frac{\partial}{\partial x} & 0 & 0 \\
0 & 0 & 0 \\
0 & 0 & \frac{\partial}{\partial z} \\
0 & \frac{\partial}{\partial x} & 0 \\
\frac{\partial}{\partial z} & 0 & \frac{\partial}{\partial x} \\
0 & \frac{\partial}{\partial z} & 0
\end{array}\right]+\left[\begin{array}{ccc}
0 & 0 & 0 \\
0 & \frac{\partial}{\partial y} & 0 \\
0 & 0 & 0 \\
\frac{\partial}{\partial y} & 0 & 0 \\
0 & 0 & 0 \\
0 & 0 & \frac{\partial}{\partial y}
\end{array}\right]=\left[D_{\Omega}\right]+\left[D_{y}\right]
$$

$D_{y}$ is the differential operator on beam axisy and $D_{\Omega}$ represent the differential operator on the cross-section of the beam. Hook's law can be used to derive the stress field by considering the elastic and the isotropic material (see Ref. [28]):

$$
\sigma=C \varepsilon
$$

\subsection{Refined one-dimensional models}

In a one-dimensional problem, the displacement field $u$ is written as the product of two contributions, a functions expansion $F_{\tau}$ over the cross-section and the unknown displacement $\mathrm{u}_{\tau}$ on the $\mathrm{y}$-axis $([29,30])$ :

$$
u=F_{\tau}(x, z) u_{\tau}(y)
$$

where the index $\tau$ ranges from 1 to the number of terms of the expansion of $\mathrm{N}$ order. The virtual variation of $\mathrm{u}$ is shown by index $\mathrm{s}$ :

$$
\delta u=F_{s}(x, z) \delta u_{s}(y)
$$

The Taylor (TE) and the Lagrange (LE) expansions were used over the cross-section. When TE models are used, the function $F_{\tau}$ is assumed to be a polynomial with a form of $x^{m}$ $z^{n}$, where $m$ and $n$ are positive integers. For instance, the displacement field of the second-order model, TE2 $(\mathrm{N}=2)$, is expressed as:

$$
\begin{aligned}
& u_{x}=u_{x_{1}}+x u_{x_{2}}+z u_{x_{3}}+x^{2} u_{x_{4}}+x z u_{x_{5}}+z^{2} u_{x_{6}} \\
& u_{y}=u_{y_{1}}+x u_{y_{2}}+z u_{y_{3}}+x^{2} u_{y_{4}}+x z u_{y_{5}}+z^{2} u_{y_{6}} \\
& u_{z}=u_{z_{1}}+x u_{z_{2}}+z u_{z_{3}}+x^{2} u_{z_{4}}+x z u_{z_{5}}+z^{2} u_{z_{6}}
\end{aligned}
$$


In this case, the functions $F_{\tau}(x, z)$ are:

$$
\begin{aligned}
& F_{1}(x, z)=1 ; \quad F_{2}(x, z)=x \quad F_{1}(x, z)=z \\
& F_{1}(x, z)=x^{2} \quad F_{1}(x, z)=x z \quad F_{1}(x, z)=z^{2}
\end{aligned}
$$

The Timoshenko beam theory (TBT) can be derived as a particular case by considering only some terms:

$$
\begin{aligned}
& u_{x}=u_{x_{1}} \\
& u_{y}=u_{y_{1}}+x u_{y_{2}}+z u_{y_{3}} \\
& u_{z}=u_{z_{1}}
\end{aligned}
$$

Considering TBT, the function expansions $\mathrm{F} \tau(\mathrm{x}, \mathrm{z})$ are:

$$
\begin{aligned}
& F_{1_{x}}=F_{1_{y}}=F_{1_{z}}=1 ; \\
& F_{2_{x}}=F_{2_{z}}=0 \quad F_{2_{y}}=x \\
& F_{3_{x}}=F_{3_{z}}=0 \quad F_{3_{y}}=z
\end{aligned}
$$

The Euler-Bernoulli beam theory (EBBT) can be derived by imposing a penalization on the shear terms (see [27]) in order to obtain: $u_{2 y}=\frac{\partial u_{x}}{\partial y}$ and $u_{3 y}=\frac{\partial u_{z}}{\partial y}$.

The LE model exploits the Lagrange polynomials to build $1 \mathrm{D}$ higher-order models. In this case the cross-section is discretized using two-dimensional Lagrange elements, and two types of elements were adopted to analyze the structures that are considered in this paper, including the four-point elements (L4) and nine-point elements (L9). The L4 interpolation functions are linear and have the following form:

$$
\begin{array}{ll}
F_{1}=\frac{1}{4}(1-r)(1-s) & F_{2}=\frac{1}{4}(1+r)(1-s) \\
F_{3}=\frac{1}{4}(1+r)(1+s) & F_{4}=\frac{1}{4}(1-r)(1+s)
\end{array}
$$

L9 provides a quadratic approximation, and the functions are:

$$
\begin{array}{cc}
F_{\tau}=\frac{1}{4}\left(r^{2}+r \cdot r_{\tau}\right)\left(s^{2}+s \cdot s_{\tau}\right) & \tau=1,3,5,7 \\
F_{\tau}=\frac{1}{2} s_{\tau}^{2}\left(s^{2}-s \cdot s_{\tau}\right)\left(1-r^{2}\right)+\frac{1}{2} r_{\tau}^{2}\left(r^{2}-r \cdot r_{\tau}\right)\left(1-s^{2}\right) & \tau=2,4,6,8 \\
F_{\tau}=\left(1-r^{2}\right)\left(1-s^{2}\right) & \tau=9
\end{array}
$$

Using LE, the unknowns are only the displacements of the cross-sectional nodes.

\subsection{Finite element approximations}

The FEM is used to approximate the solution along the beam axis. If the shape functions $\mathrm{N}_{\mathrm{i}}$ are introduced, the unknown displacement $u_{\tau}(y)$ becomes:

$$
u_{\tau}(y)=N_{i}(y) q_{i \tau}
$$

where qi $\tau$ are the unknowns of the problem. The virtual variation of $u_{\tau}(y)$ is denoted using the index $\mathrm{j}$ :

$$
\delta u_{s}(y)=N_{j}(y) \delta q_{j s}
$$

Finally, the complete displacement field and its virtual variation can be written as follows:

$$
\begin{aligned}
& u(y)=F_{\tau} N_{i}(y) q_{i \tau} \\
& \delta u(y)=F_{s} N_{j}(y) \delta q_{j s}
\end{aligned}
$$

where indexes $i$ and $j$ indicate the node of the element along the y-axis. In this work, three- or four-node refined beam elements were used on the $y$-axis.

\subsection{Governing Equations}

The governing equation can be derived using the Principle of Virtual Displacements (PVD) that assumes the following form in the case of the free vibration analysis:

$$
\delta L_{\text {int }}+\delta L_{\text {ine }}=0
$$

where $L_{i n t}$ stands for the strain energy and $L_{i n e}$ is the inertial work. $\delta$ stands for the virtual variation. $L_{i n t}$ can be written as:

$$
\delta L_{\text {int }}=\int_{V} \delta \varepsilon^{T} \sigma d V
$$

By introducing the stress and strain given in Equations 2 and 3 as well as in Equations 19 and 20, the variation of the internal work becomes:

$$
\delta L_{\text {int }}=\int_{V} \delta q_{j s}^{T}\left[N_{j}(y) F_{s}(x, z) D^{T} C D F_{\tau}(x, z) N_{i}(y)\right] q_{i \tau} d V
$$

By splitting the integral on the volume $V$ into two subintegrals, one on the cross-section $\Omega$ and one along the beam axis $l$, Equation 23 becomes:

$$
\begin{aligned}
& \delta L_{\text {int }}=\delta q_{j s}^{T}\left\{\int_{V}\left[\left(D_{\Omega}+D_{y}\right)^{T} F_{s}(x, z) N_{j}(y)\right] C\left[\left(D_{\Omega}+D_{y}\right) N_{i}(y) F_{\tau}(x, z)\right] d V\right\} q_{i \tau} \\
&=\delta q_{j s}^{T}\left\{\int_{l} N_{j}(y)\left[\int_{\Omega} D_{\Omega}{ }^{T} F_{s}(x, z) C D_{\Omega} F_{z}(x, z) d \Omega\right] N_{i}(y) d y\right. \\
& \\
&+\int_{l} N_{j}(y)\left[\int_{\Omega} D_{\Omega}^{T} F_{s}(x, z) C F_{\tau}(x, z) d \Omega\right] D_{y} N_{i}(y) d y \\
&+\int_{l} D_{y}^{T} N_{j}(y)\left[\int_{\Omega} F_{s}(x, z) C D_{\Omega} F_{\tau}(x, z) d \Omega\right] N_{i}(y) d y \\
&\left.+\int_{l} D_{y}^{T} N_{j}(y)\left[\int_{\Omega} F_{s}(x, z) C F_{\tau}(x, z) d \Omega\right] D_{y} N_{i}(y) d y\right\} q_{i \tau}
\end{aligned}
$$

Where $K^{i j t s}$ is the stiffness matrix in the form of the fundamental nucleus that is always characterized by a $3 \times 3$ matrix. The explicit form of the fundamental nucleus can be 
found in Ref. [28].

The virtual variation of the $L_{\text {ine }}$ is:

$$
\delta L_{\text {ine }}=\int_{V} \delta u^{T} \rho \ddot{u} d V
$$

where $\rho$ is the density of the material and $\ddot{u}$ is the acceleration vector. Equation 25 can be rewritten using Equations 4 and 17:

$$
\delta L_{\text {ine }}=\int_{l} \delta q_{j s}^{T} N_{j}(y)\left\{\int_{\Omega} F_{s}(x, z) \rho F_{\tau}(x, z) d \Omega\right\} N_{i}(y) \ddot{q}_{i \tau} d y=\delta q_{j s}^{T} M^{i j \pi} \ddot{q}_{i \tau}
$$

where the mass matrix $M^{i j t s}$ is a $3 \times 3$ fundamental nucleus, and the indexes have the same meaning as that in the stiffness matrix. The global stiffness $(K)$ and mass $(M)$ matrices can be obtained assembling the fundamental nucleus evaluated for each combination of the indices. In conclusion, the PVD can be written as follows:

$$
\delta q(M \ddot{q}+K q)=0
$$

where $q$ is the vector of the nodal unknowns of the whole structure. Due to the linearity of the problem where harmonic solutions are introduced, it is possible to compute the natural frequencies $\left(\omega_{k}\right)$ by solving the following eigenvalues problem:

$$
\left(-\omega_{k}^{2} M+K\right) q_{k}=0
$$

Where $q_{k}$ is the $k$-th eigenvector, and $k$ ranges form 1 to the total number of DOFs of the structures.

\subsection{Matrices Assembly}

The previous sections shows that the use of the CUF allows the matrices to be written in terms of the fundamental nuclei, and these $3 \times 3$ matrices can be used to build the global stiffness and mass matrices. Fig. 5 shows the assembly procedure. The assembly of the matrices requires the use of

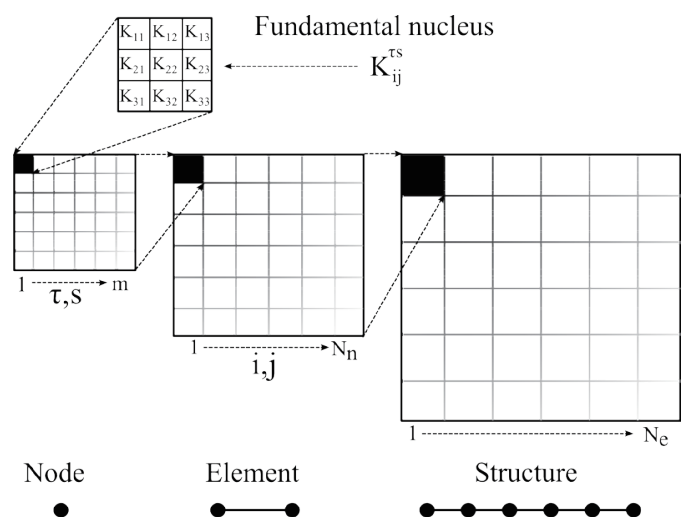

Fig. 5. Fundamental nuclei assembly procedure. five loops. The first loop is of the element number. Once the element is fixed, two loops on indexes $i$ and $j$ are required, and these indexes are related to the node of the beam element. Finally, given $i, j$ and the element number, it is possible to perform two loops on indexes $\tau$ and $\mathrm{s}$ to represent the expansion over the cross-section.

Calculating the matrices requires the fundamental nucleus to be evaluated for each index combination. This procedure can easily take advantage of the parallel processing to reduce the computational time. The matrices that are obtained are symmetric banded matrices, and this problem can be solved using efficient techniques that reduce the computational time. The bandwidth depends on the number of terms used in the function expansion that is used over the cross-section and by the number of nodes for each beam element. The more refined that the beam model is, the higher the bandwidth.

\section{Numerical results}

This section investigates the capabilities of different beam models in the free vibration analysis of thin-walled stiffened structures. Three structures are considered, including:

- a stiffened plate;

- a stiffened cylinder with eight longitudinal stringers and one transversal rib;

- a launcher model that includes both longitudinal and transversal stiffeners with two lateral boosters.

TE and LE expansions are used, and a different order of expansion $(\mathrm{N})$ is considered when TE models are used while different meshes over the cross-section are taken into account when LE models are considered. The results obtained by the TE and LE models are compared to those obtained using the MSC NASTRAN commercial code. Two approaches were used: the first considers a 3D model, and the second uses $1 \mathrm{D}$ and 2D models joined together. The structures are built using aluminum with a Young modulus $(E)$ of $75 \mathrm{GPa}$, Poisson ratio ( $v$ ) of 0.3 and density $(\rho)$ equal to $2700 \mathrm{~kg} / \mathrm{m}^{3}$.

\subsection{Assessment and convergence analysis}

A stiffened plate was considered in order to assess the model and to perform some convergence analyses. The geometry of the model is reported in Fig. 6. The structure is clamped at $\mathrm{y}=0$ and $\mathrm{y}=10 \mathrm{~m}$. The assessments focused on the effects of the choice of the expansion over the cross-section. TE models with a different order were considered, and when the LE model was used, different discretizations over the 
cross-section were taken into account. Fig. 7 shows some of the cross-sectional approximations that were considered. The convergence analysis was conducted by increasing the number of elements used for each panel between the reinforcements, and the notation LE-(N)E was used when (N) elements were used between the reinforcements. In the case of the TE models, the convergence was investigated with an increasing order of expansion. TE(N) denotes a model of order $(\mathrm{N})$, and EULE denotes the Euler-Bernoulli model. Five cubic beam elements were thus used along the beam axis.

Table 1 shows the first five frequencies that were evaluated using different models. The reference results were been obtained using a full 3D FEM model. The results show that the TE models require a very low number of DOFs but, a sixth order model, TE6, still does not provide good convergence.

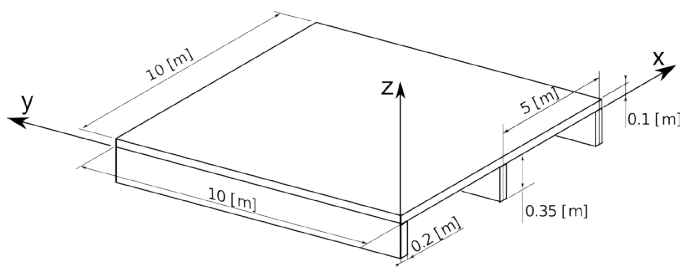

Fig. 6. Reinforced plate geometry.

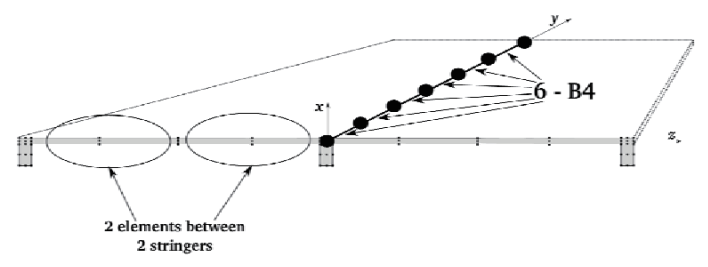

(a) LE-2E Model
Moreover, some modes cannot be detected by using lower order models, and the LE models provide better results. Convergence can be easily achieved by improving the discretization over the cross-section. When 3 elements are used in between the two reinforcements in the LE-3E model, the results are accurate and the number of DOFs is just one half that of the reference model. It is clear that the modes with a higher number of half-waves require a refined mesh in order to be accurately evaluated. The convergence behavior of the first five modes is shown in Fig. 8. From these results, it is clear that the LE models are able to detect very complex modal shapes, and the results converge to the reference values. On the other hand, TE models are very cheap but do not provide accurate results for reinforced structures.

\subsection{Reinforced cylinder}

The geometry of the cylindrical structure is shown in Fig. 9. It has length $\mathrm{L}$ that is equal to $15 \mathrm{~m}$ and has both ends clamped. Three components were used to build the entire structure. Fig. 10 shows the beam configuration, where components 1 and 3 have the same cross-section (Fig. 11a). Components 1 and 3 are thin-walled cylinders reinforced with eight stringers while component 2 (Fig. 11b)

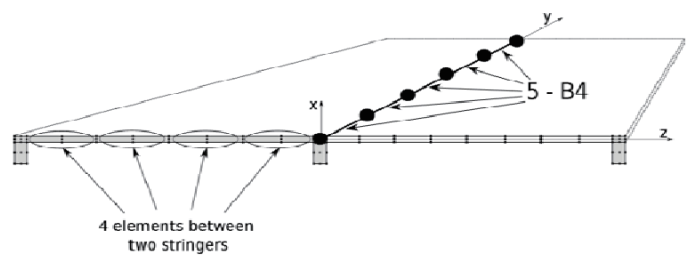

(b) LE-4E Model

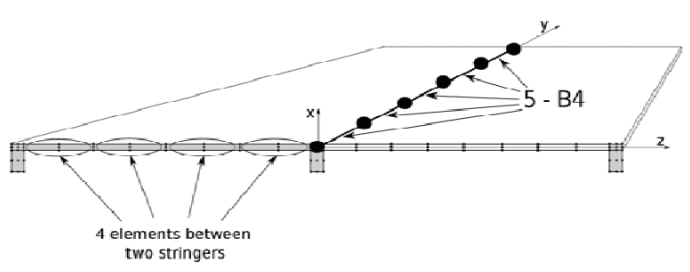

(c) LE-6E Model

Fig. 7. Reinforced panel LE models: example of different cross-sectional disctretizations

Table 1. First 5 natural frequencies of the reinforced plate evaluated using TE and LE models

\begin{tabular}{|c|c|c|c|c|c|c|c|c|c|c|c|c|c|}
\hline & FEM3D & LE-2E & LE-3E & LE-4E & LE-5E & LE-6E & EULE & TE1 & TE2 & TE3 & TE4 & TE5 & TE6 \\
\hline DOFs & 6840 & 3024 & 3600 & 4176 & 4752 & 5328 & 48 & 144 & 288 & 480 & 720 & 1008 & 1344 \\
\hline 1 & 14.1 & 15.0 & 14.5 & 14.3 & 14.2 & 14.1 & 21.2 & 21.0 & 18.5 & 18.2 & 17.8 & 17.6 & 16.4 \\
\hline 2 & 14.5 & 15.4 & 14.9 & 14.8 & 14.7 & 14.6 & - & 161.2 & 27.3 & 23.3 & 22.7 & 20.8 & 19.7 \\
\hline 3 & 23.6 & 24.0 & 23.9 & 23.7 & 23.7 & 23.6 & - & - & 156.0 & 42.2 & 36.8 & 31.7 & 29.3 \\
\hline 4 & 27.2 & 30.1 & 28.3 & 27.8 & 27.6 & 27.5 & - & - & 71.0 & 50.9 & 54.7 & 49.0 & 42.6 \\
\hline 5 & 28.1 & 29.5 & 28.7 & 28.4 & 28.2 & 28.2 & ${ }_{-}^{*}$ & - & ${ }^{*}$ & 164.4 & 90.1 & 45.3 & 34.4 \\
\hline
\end{tabular}


Erasmo Carrera Accurate Free Vibration Analysis of Launcher Structures Using Refined 1D Models

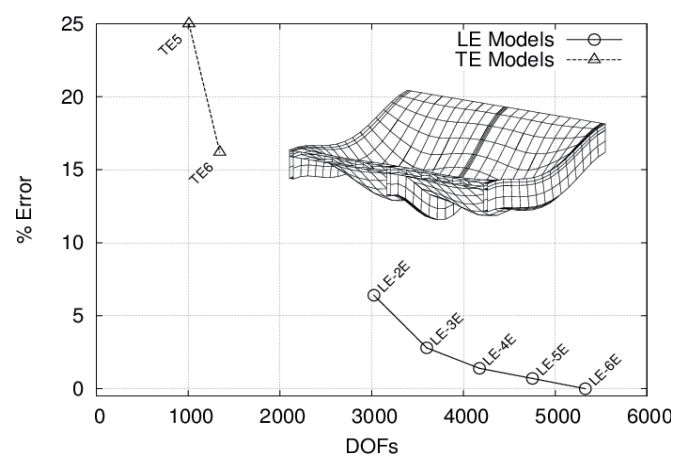

(a) Mode $1,14.1 \mathrm{~Hz}$

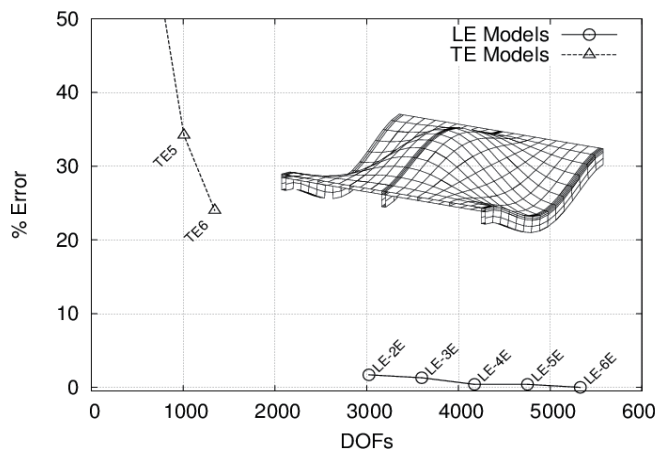

(c) Mode $3,23.6 \mathrm{~Hz}$

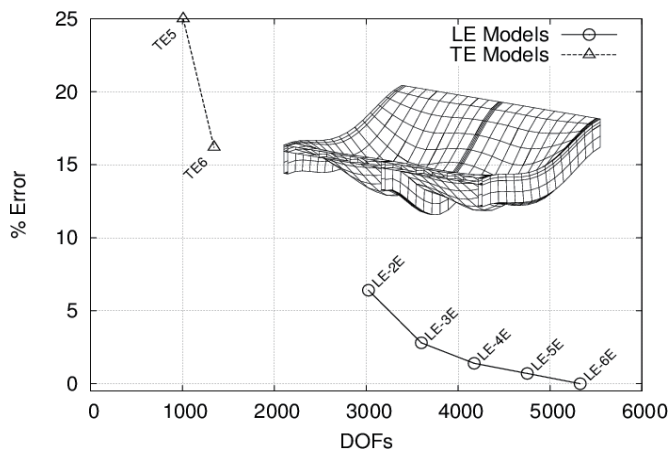

(b) Mode $2,14.5 \mathrm{~Hz}$

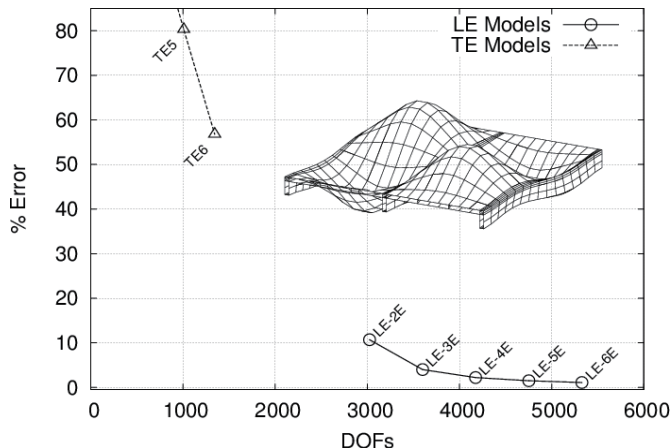

(d) Mode 4, 27.2 Hz

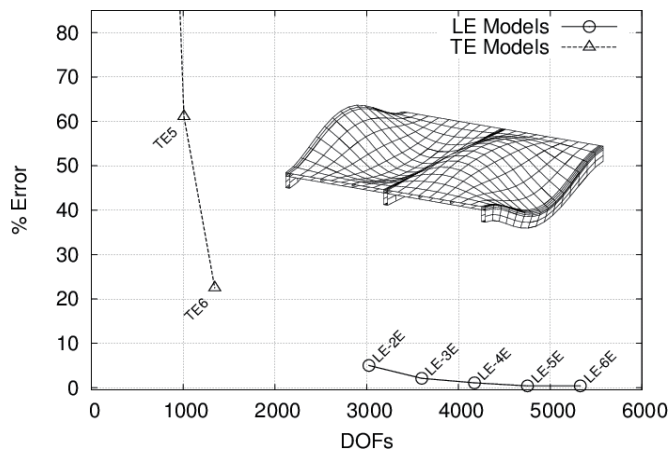

(e) Mode 5, $28.1 \mathrm{~Hz}$

Fig. 8. Convergence analysis of the reinforced panel.

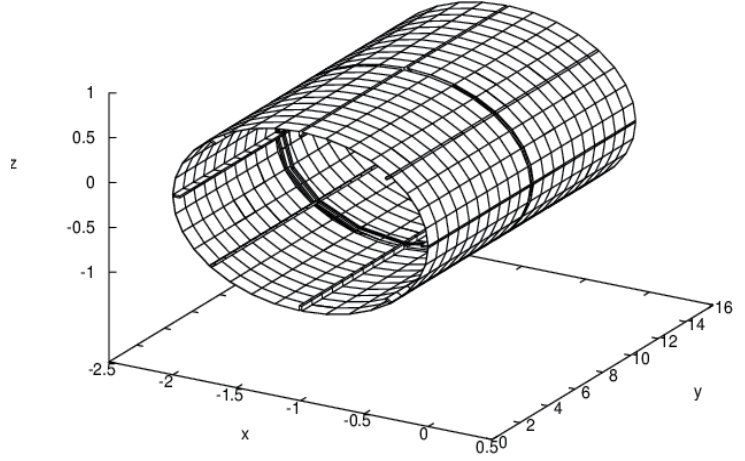

Fig. 9. Reinforced cylinder model.

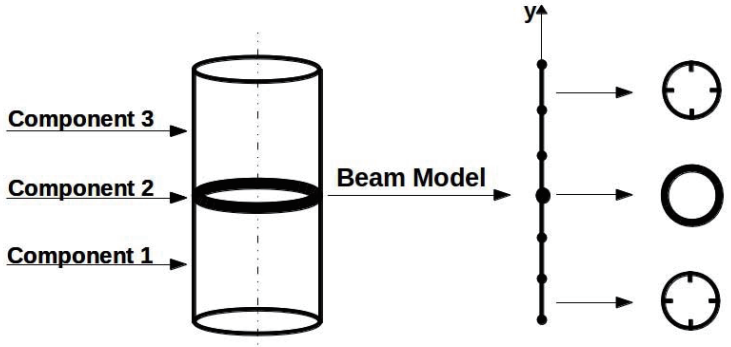

Fig. 10. Component-wise representation of the reinforced cylinder. 
is a circumferential reinforcement or rib. The geometrical properties of both components are shown in Fig. 11.

The aim of this modal analysis is to highlight the

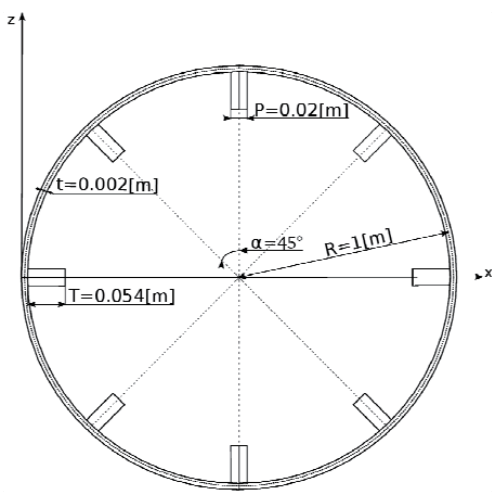

(a) Components 1 and 3

Fig. 11. Reinforced cylinder cross-section geometry.

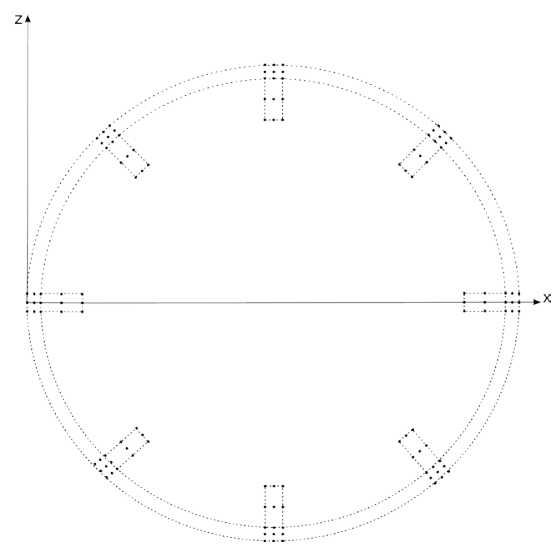

Fig. 12. LE distretization of the cross-section of the reinforced cylinder. capabilities of the TE/LE model in evaluating the classical beam modes, such as bending and torsional modes. The results are compared to those obtained using two FE models

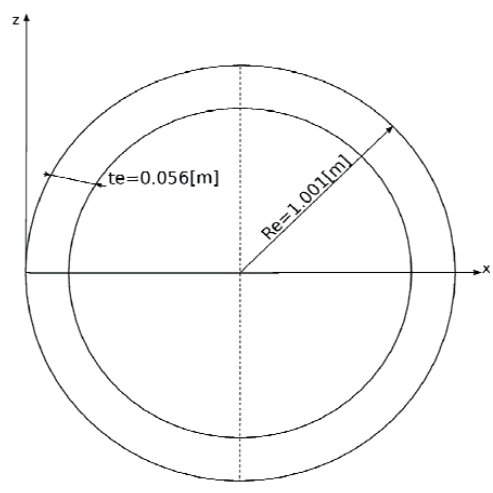

(b) Components 2

built using the MSC NASTRAN commercial code. The 3D FE model was built using solid elements while for the $2 \mathrm{D}-1 \mathrm{D}$ FE model, shell elements are used to simulate the skin while beam elements are used for both the stringers and the rib with the correct offset from skin included. When TE models are used, different $\mathrm{N}$ orders of expansion are considered to evaluate the convergence to the reference solution. The LE model is built using 24 nine-node elements over the crosssection, as shown in Fig. 12. When LE models are used, two different discretizations are considered along the y-axis. The former considers 4 beam elements with 3 nodes (beam-3) for components 1 and 3 while the latter has 8 cubic elements for the same components. For both LE models, component 2

Table 2. First 15 frequencies of the reinforced cylinder evaluated using the TE models

\begin{tabular}{cccccc}
\hline$f_{S}[\mathrm{~Hz}]$ & $T E 1$ & $T E 2$ & $T E 3$ & $T E 4$ & $T E 5$ \\
\hline 1 & $42.25^{(B)}$ & $42.90^{(B)}$ & $38.98^{(B)}$ & $38.92^{(B)}$ & $38.79^{(B)}$ \\
2 & $42.25^{(B)}$ & $42.90^{(B)}$ & $39.04^{(B)}$ & $38.97^{(B)}$ & $38.94^{(B)}$ \\
3 & $91.53^{(T)}$ & $91.53^{(T)}$ & $78.01^{(S)}$ & $70.05^{(S)}$ & $53.24^{(S)}$ \\
4 & $120.66^{(A)}$ & $123.04^{(B)}$ & $78.09^{(S)}$ & $70.14^{(S)}$ & $53.28^{(S)}$ \\
5 & $121.95^{(B)}$ & $123.05^{(B)}$ & $91.53^{(T)}$ & $83.28^{(S)}$ & $67.21^{(S)}$ \\
6 & $121.95^{(B)}$ & $147.57^{(A)}$ & $107.50^{(B)}$ & $83.29^{(S)}$ & $70.47^{(S)}$ \\
7 & $193.06^{(B)}$ & $195.24^{(B)}$ & $107.71^{(B)}$ & $91.53^{(T)}$ & $70.48^{(S)}$ \\
8 & $193.06^{(B)}$ & $195.24^{(B)}$ & $120.87^{(S)}$ & $102.77^{(S)}$ & $77.27^{(S)}$ \\
9 & $220.66^{(T)}$ & $220.66^{(T)}$ & $120.88^{(S)}$ & $102.78^{(S)}$ & $91.53^{(T)}$ \\
10 & $281.81^{(T)}$ & $281.81^{(T)}$ & $147.33^{(A)}$ & $107.27^{(B)}$ & $92.78^{(S)}$ \\
11 & $295.96^{(A)}$ & $307.28^{(B)}$ & $162.12^{(B)}$ & $107.43^{(B)}$ & $92.80^{(S)}$ \\
12 & $305.67^{(B)}$ & $307.29^{(B)}$ & $162.46^{(B)}$ & $147.28^{(A)}$ & $107.18^{(B)}$ \\
13 & $305.68^{(B)}$ & $357.06^{(A)}$ & $162.66^{(S)}$ & $147.84^{(S)}$ & $107.36^{(B)}$ \\
14 & $373.53^{(A)}$ & $379.54^{(B)}$ & $162.67^{(S)}$ & $147.88^{(S)}$ & $133.75^{(S)}$ \\
15 & $377.38^{(B)}$ & $379.55^{(B)}$ & $220.63^{(T)}$ & $161.43^{(B)}$ & $139.39^{(S)}$ \\
\hline (B) Bending,(T) Torsion, $(S)$ Shell Like,(A) Assial & & &
\end{tabular}


has 1 cubic beam element along the axis. Table 2 shows the first 15 frequencies that were evaluated using different TE models. When T E1 and T E2 are considered, only bending, axial and torsional modes are found because a coarse kinematic model was used. When the N-order increases, the model is able to identify more shell-like modes, and the bending and torsional modes converge to a 3D FE solution. Table 3 shows the first 15 frequencies with both LE24 models. The model with more elements over the axis, LE24+, shows more shell-like modes before the first bending frequency with respect to LE24, and the bending frequency slightly decreases, as expected.

Table 4 shows the first two bending and torsional

Table 3. First 15 frequencies of the reinforced cylinder evaluated using the LE models

\begin{tabular}{ccc}
\hline$f_{S}[\mathrm{~Hz}]$ & $L E 24$ & $L E 24+$ \\
\hline 1 & $18.51^{(S)}$ & $17.43^{(S)}$ \\
2 & $18.67^{(S)}$ & $17.46^{(S)}$ \\
3 & $26.22^{(S)}$ & $25.10^{(S)}$ \\
4 & $26.24^{(S)}$ & $25.11^{(S)}$ \\
5 & $30.74^{(S)}$ & $30.26^{(S)}$ \\
6 & $30.80^{(S)}$ & $30.34^{(S)}$ \\
7 & $31.63^{(S)}$ & $30.48^{(S)}$ \\
8 & $31.89^{(S)}$ & $30.55^{(S)}$ \\
9 & $34.17^{(B)}$ & $31.66^{(S)}$ \\
10 & $34.55^{(B)}$ & $31.74^{(S)}$ \\
11 & $35.93^{(S)}$ & $33.99^{(B)}$ \\
12 & $36.45^{(S)}$ & $34.14^{(B)}$ \\
13 & $43.39^{(S)}$ & $42.35^{(S)}$ \\
14 & $43.51^{(S)}$ & $42.38^{(S)}$ \\
15 & $68.64^{(S)}$ & $46.71^{(S)}$ \\
\hline$(B)$ Bending, (S) Shell Like &
\end{tabular}

frequencies that were computed using different structural models. The first column reports the results evaluated using the solid model, and the accurate mesh makes these results accurate, and so these are used as reference. The 2D - 1D FE model allows the number of DOFs to be drastically reduced, but an error greater than $10 \%$ is observed in the case of the first bending and torsional frequencies. When a T E5 model is used, the bending frequencies show an error close to $15 \%$, but the error increases up to $30 \%$ if the torsional frequencies are considered. The LE models provide results that are very close to the reference solution. Both models that are proposed provide an error close to $1 \%$ in the case of the first bending and torsional frequency, and the error increases up to $6 \%$ when the second frequency is considered. Among the models that are considered, refined one-dimensional LE models appear to be more accurate. These models provide an error with respect to the solid model, but a reduction should be considered in terms of the number of DOFs. The DOFs of the LE2 4 are about $2 \%$ that of the 3D FE DOFs while the DOFs of LE24+ are $4 \%$ of that. When the axial discretization is refined, a slow convergence can be seen with respect to the 3D FE solution, as shown in Table 4.

Figure 13 shows the first bending mode for all models, including 3D, 2D - 1D, T E5 and LE24+. When both models obtained with commercial code are considered, both the global and local effects are well detected. On the other hand, only global modes appear with TE and LE models. In the case of the TE models, theses result can be improved by increasing the order of expansion $\mathrm{N}$ while for the LE models, a refined cross-sectional mesh is required to detect the local effects. Figs. 14, 15 and 16 show that both the TE and LE

Table 4. Comparisons of the first two bending and torsional frequencies of the reinforced cylinder using different structural model [Hz].

\begin{tabular}{cccccc}
\hline Mode & FEM $_{3 D}$ & FEM $_{2 D-1 D}$ & TE 5 & LE 24 & LE 24+ \\
\hline DOF: & 390192 & 26206 & 2142 & 8352 & 15264 \\
\hline Bending & Frequencies: & & & & \\
\hline $1^{a}$ & 33.64 & $37.49^{(+11.4 \%)}$ & $38.79^{(+15.3 \%)}$ & $34.17^{(+1.6 \%)}$ & $33.99^{(+1.0 \%)}$ \\
$2^{a}$ & 94.82 & $91.06^{(-4.0 \%)}$ & $107.18^{(+13.0 \%)}$ & $88.89^{(-6.3 \%)}$ & $89.07^{(-6.4 \%)}$ \\
\hline Torsion & Frequencies: & & & \\
\hline $1^{a}$ & 67.67 & $77.83^{(+15.0 \%)}$ & $91.53^{(+35.3 \%)}$ & $68.65^{(+1.4 \%)}$ & $68.32^{(+0.9 \%)}$ \\
$2^{a}$ & 175.33 & $179.49^{(+2.4 \%)}$ & $220.63^{(+25.8 \%)}$ & $163.90^{(-6.5 \%)}$ & $162.90^{(-7.6 \%)}$ \\
\hline
\end{tabular}

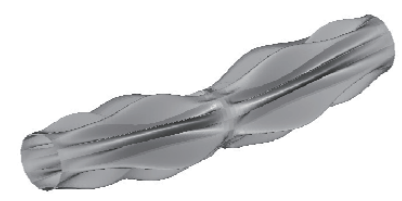

(a) $f_{3 D}=33.64 \mathrm{~Hz}$

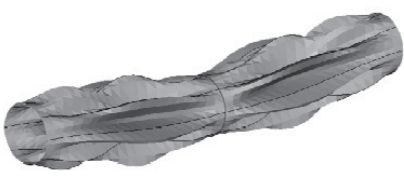

(b) $f_{2 D-1 D}=37.49 H z$

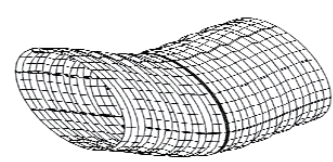

(c) $\mathrm{f}_{\mathrm{TE} 5}=38.79 \mathrm{~Hz}$

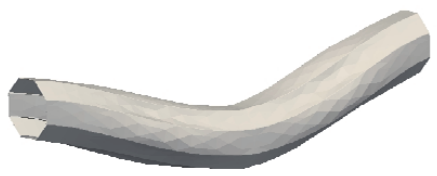

(d) $\mathrm{f}_{\text {LE24+ }}=33.99 \mathrm{~Hz}$

Fig. 13. First bending mode of the reinforce cylinder. 


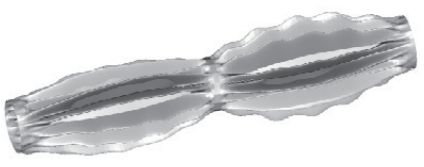

(a) $f_{3 D}=94.82 \mathrm{~Hz}$

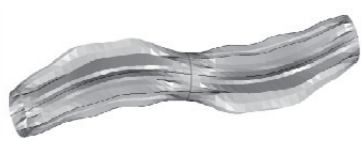

(b) $f_{2 D-1 D}=91.07 \mathrm{~Hz}$

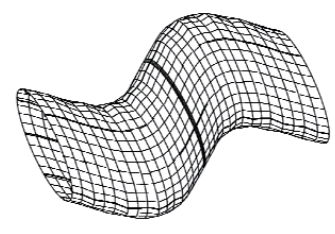

(c) $f_{\text {TE5 }}=107.18 \mathrm{~Hz}$

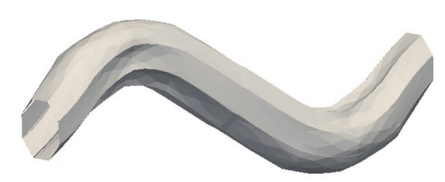

(d) $\mathrm{fLE}_{\mathrm{L} 2+}=89.07 \mathrm{~Hz}$

Fig. 14. Second bending mode of the reinforce cylinder.

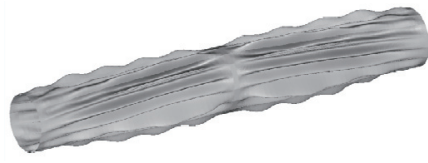

(a) $f_{3 D}=67.67 \mathrm{~Hz}$

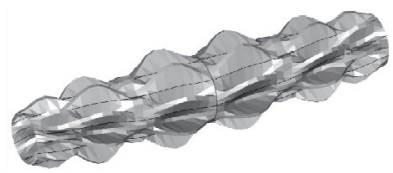

(b) $f_{2 D-1 D}=77.83 \mathrm{~Hz}$

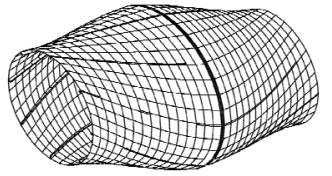

(c) $\mathrm{f}_{\mathrm{TES}}=91.53 \mathrm{~Hz}$

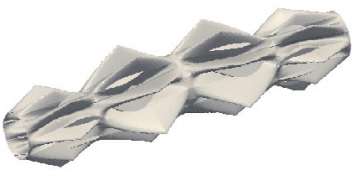

(d) $\mathrm{fLE}_{\mathrm{L} 4+}=68.32 \mathrm{~Hz}$

Fig. 15. First torsional mode of the reinforce cylinder.

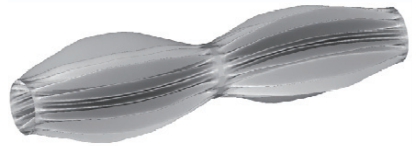

(a) $f_{3 D}=175.33 \mathrm{~Hz}$

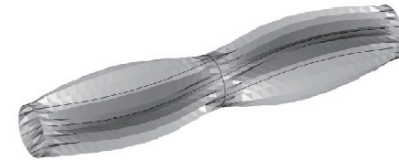

(b) $f_{2 D-1 D}=179.49 H z$

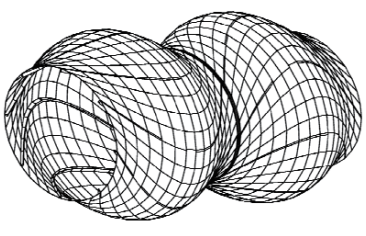

(c) $f_{\text {TE5 }}=220.63 \mathrm{~Hz}$

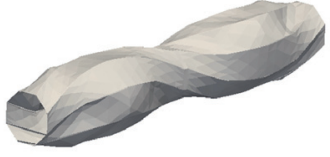

(d) $\mathrm{f}_{\text {LE24+ }}=162.90 \mathrm{~Hz}$

Fig. 16. Second torsional mode of the reinforce cylinder.

models are able to accurately detect the global behaviour shown by the 3D FE model. In conclusion, an analysis of the results suggests the following:

-When TE models are used, many terms in the expansion are required in order to ensure good agreement with the commercial code in detecting shell-like modes;

- LE models provide good result using fewer DOFs than both 2D - 1D and 3D FE models;

- Reinforced thin-walled structures require an LE model for proper analysis.

\subsection{Complete launcher modal analysis}

The geometry of the complete schema of the launcher that is considered in this work is shown in Fig. 17. The entire structure is composed of three main components, a central body and two lateral boosters that are attached to the central body via four connection points CP. Eleven components are used to build this launcher structure, as shown in Fig. 18. This Fig. shows that the launcher can be considered as one beam with a variable section. Fig. 19 shows the 7 different cross-sections that are used to build the model. The circumferential reinforcements are reported in Figs. 19a, e, and $g$ and are respectively used in components 1, 5 and 9.
The components 2, 4, 6, 8 and 10 are thin-walled cylinders with four longitudinal reinforcements, and these use the sections that are shown in Figs. 19b, d and f. Fig. 19c shows

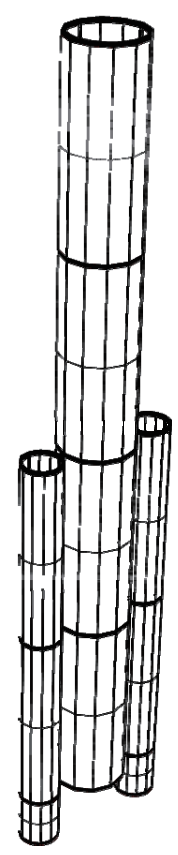

Fig. 17. Launcher configuration. 
the section that was used for components 3 and 7 where the central body is connected with the boosters. The geometrical dimensions of these 7 cross-sections are shown in Table 5. The components that include a transversal reinforcement have only one cubic element along the axis while the others, where there is a thin-walled configuration, have two cubic elements. Both stringers and CP properties are reported in Table 6. A detailed view of the discretization of section 3 is reported in Fig. 20. When the LE models are considered, the position of the cross-sectional nodes is influenced by the geometry, and in this case, it is important to place these in the correct position connect the central body with the booster.

The results are compared with those from two FE models that were built using the MSC NASTRAN commercial code. The first model is built using solid elements while the shell and beam elements compose the second FE model. The shell elements are used to simulate skin while the beam elements

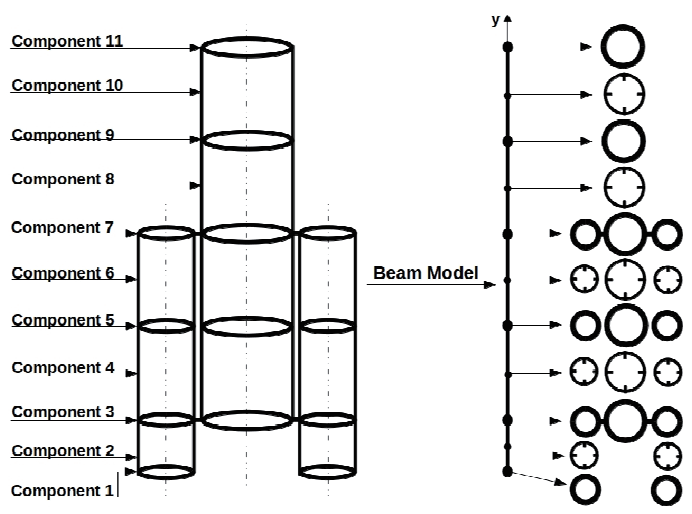

Fig. 18. Component-wise representation of the launcher. are used for stringers and ribs. No constraints were imposed on the model, but the null frequencies have been neglected in the results due to the rigid body motion. Table 7 shows the first 15 frequencies that were evaluated using the TE models. When $\mathrm{N}$ increases, the TE models can identify more complex modes. Table 8 shows the first 25 frequencies that were evaluated with the Lagrange, LE, model and with the FE models created using the commercial code. The results with LE are very close to the results obtained with the Solid FE model, but this model has only $5 \%$ of the DOFs with respect to the Solid model while the $2 \mathrm{D}-1 \mathrm{D}$ model has $72 \%$ of the DOFs.

Table 9 shows 12 frequencies that were chosen among the frequencies reported in Table 8 , and these are used to compare the results in terms of the modes. While Table 8 shows the first 25 frequencies of each model without any considerations of the modal shape, the frequencies in Table 9 correspond to the same mode. The results obtained with the T E3 model show that 10 terms in the expansion were not sufficient to find an accurate solution. Even if, the DOFs were only $0.23 \%$ of the DOFs of the reference model, the third order model based on the Taylor expansion should not be considered to be accurate in the analysis of such structures. The results obtained using the LE, 1D - 2D and the Solid models provide complex modes that may involve the entire structure or only a part of it. When the LE model is used, all the 12 reference modes are found while the use of the $2 \mathrm{D}-1 \mathrm{D}$ FE model is missing mode 5 . The fourth reference mode, which is shown in Fig. 21, is a complex mode where a local bending mode characterizes the central body while the lateral bodies are characterized by a shell-

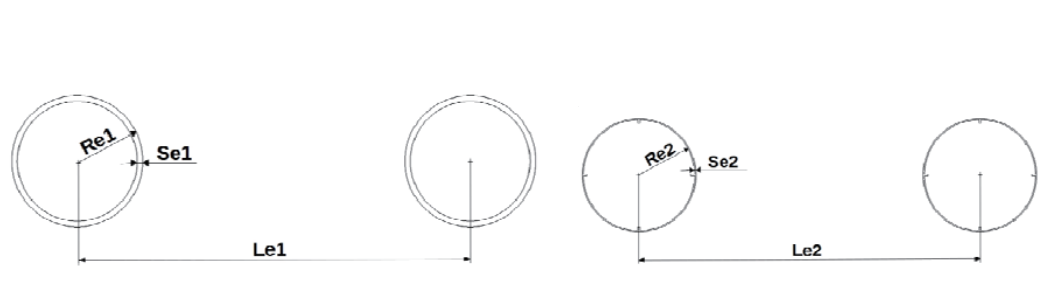

(a) Section 1

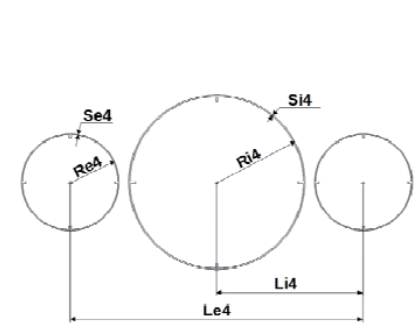

(d) Section 4 (b) Section 2

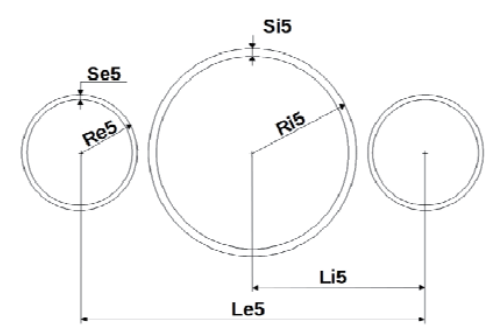

(e) Section 5

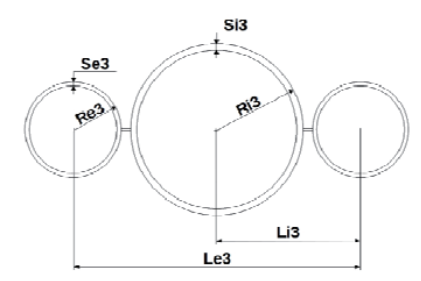

(c) Section 3

Fig. 19. Cross-section geometry of the launcher components. 
like mode. In this case, the same error is committed if both the 2D - 1D FE model and the LE model are compared with the Solid model. The reference mode 6 , which is shown in Fig. 22, is a global bending mode that involves a complete structure. For this mode, the frequency that is evaluated with the LE model is more accurate than that from the $1 \mathrm{D}-2 \mathrm{D}$ model. The reference mode 9 (Fig. 23) is more accurate if it is evaluated using the $2 \mathrm{D}-1 \mathrm{D}$ model while both LE9 and

Table 5. Geometrical data of the components of the launcher

\begin{tabular}{|c|c|c|c|c|}
\hline ID Sec & Comp & $N_{B E A M}$ & Cross-Section $[\mathrm{m}]$ & Lenght $[\mathrm{m}]$ \\
\hline 1 & 1 & 1 & $\begin{array}{l}R e_{1}=1.50 \\
S e_{1}=0.13 \\
L e_{1}=9.00\end{array}$ & $h=0.25$ \\
\hline 2 & 2 & 2 & $\begin{array}{l}R e_{2}=1.50 \\
S e_{2}=0.03 \\
L e_{2}=9.00\end{array}$ & $h=3.50$ \\
\hline 3 & $3 / 7$ & 1 & $\begin{aligned} \mathrm{Re}_{3} & =1.50 \mathrm{Ri}_{3}=2.70 \\
\mathrm{Se}_{3} & =0.13 \mathrm{Si}_{3}=0.20 \\
\mathrm{Le}_{3} & =9.00 \mathrm{Li}_{3}=4.50\end{aligned}$ & $h=0.25$ \\
\hline 4 & $4 / 6$ & 2 & $\begin{array}{l}\mathrm{Re}_{4}=1.50 \mathrm{Ri}_{4}=2.70 \\
\mathrm{Se}_{4}=0.03 \mathrm{Si}_{4}=0.04 \\
\mathrm{Le}_{4}=9.00 \mathrm{Li}_{4}=4.50\end{array}$ & $h=13.50$ \\
\hline 5 & 5 & 1 & $\begin{aligned} R e_{5} & =1.50 R i_{5}=2.70 \\
S e_{5} & =0.13 S i_{5}=0.20 \\
L e_{5} & =9.00 L_{5}=4.50\end{aligned}$ & $h=0.25$ \\
\hline 6 & $8 / 10$ & 2 & $\begin{array}{l}R i_{6}=2.70 \\
S i_{6}=0.04\end{array}$ & $h=13.50$ \\
\hline 7 & $9 / 11$ & 1 & $\begin{array}{l}R i_{7}=2.70 \\
S i_{7}=0.20\end{array}$ & $h=0.25$ \\
\hline
\end{tabular}

Table 6. Geometries of the cross-sections of the launcher components

\begin{tabular}{lccc}
\hline Dimensions & $S_{i}$ & $S_{e}$ & $C P$ \\
\hline Long side & 0.16 & 0.10 & 0.30 \\
Short side & 0.06 & 0.06 & 0.06 \\
\hline$S_{i}:$ Stringers located in the central body & \\
$S_{e}:$ Stringers located in the lateral bodies \\
$C P:$ Connection Points
\end{tabular}

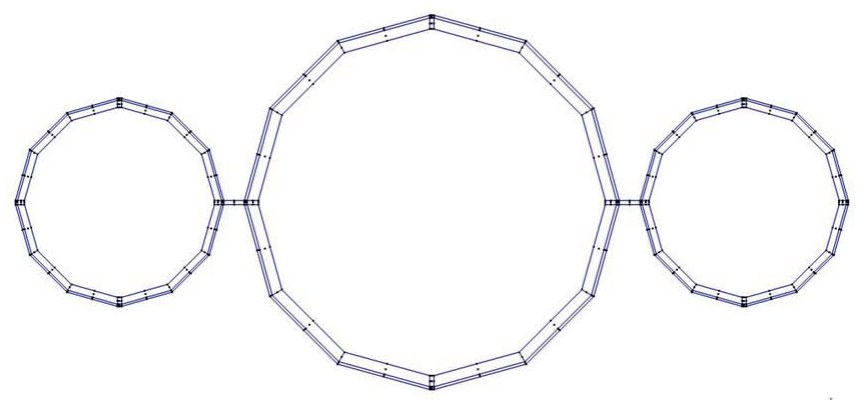

(a) Overall Section the $2 \mathrm{D}$ - 1D models commit the same error when evaluating mode 10 (Fig. 24). Modes 11 and 12 (Figs. 25 and 26) exhibit quite complex modal shapes, and the results from the LE model are accurate. The results shown in Table 9 highlight that, even if the results evaluated using the LE still have some errors, these errors are often lower than those provided

Fig. 20. LE discretization of the joint between the central body and the lateral boosters. 
by the $1 \mathrm{D}-2 \mathrm{D}$ model. At the same time, the LE model drastically reduces the number of DOFs with respect to the models built using the commercial code. In fact, this model only has $5 \%$ of the DOFs with respect to the solid model and $7 \%$ with respect to the $1 \mathrm{D}-2 \mathrm{D}$ model. The computational efficiency and high level of accuracy of the LE model, which is are at least comparable to those of the 1D - 2D model, make the refined one-dimensional model very attractive in the dynamic analysis of complex structures, as for the launcher that is considered here.

\section{Concluding Remarks}

In this paper, different finite elements models were compared for the free vibration analysis of thin-walled

Table 7. First 15 frequencies of the launchers evaluated using the TE models

\begin{tabular}{cccc}
\hline$f_{S}[\mathrm{~Hz}]$ & $T E 2$ & $T E 3$ & $T E 4$ \\
\hline DOF & 414 & 1290 & 1935 \\
\hline 1 & 8.73 & 7.57 & 8.29 \\
2 & 11.78 & 9.94 & 9.00 \\
3 & 15.17 & 13.29 & 10.20 \\
4 & 16.74 & 14.65 & 11.42 \\
5 & 20.67 & 14.96 & 12.68 \\
6 & 22.53 & 16.18 & 20.39 \\
7 & 27.30 & 19.52 & 24.86 \\
8 & 33.20 & 20.81 & 25.61 \\
9 & 37.35 & 23.34 & 28.58 \\
10 & 39.43 & 25.28 & 33.51 \\
11 & 46.19 & 26.34 & 40.40 \\
12 & 46.93 & 29.65 & 43.77 \\
13 & 48.21 & 33.27 & 45.49 \\
14 & 52.76 & 33.35 & 48.08 \\
15 & 53.35 & 36.74 & 49.21 \\
\hline
\end{tabular}

Table 8. First 25 frequencies of the launchers evaluated using the LE, the 1D-2D and the Solid models

\begin{tabular}{cccc}
\hline$f_{S}[\mathrm{~Hz}]$ & $F E M_{3 D}$ & $F E M_{2 D-1 D}$ & $L E 9$ \\
\hline DOF & 565740 & 408456 & 29682 \\
\hline 1 & 0.54 & 0.46 & 0.74 \\
2 & 0.63 & 0.56 & 0.92 \\
3 & 3.76 & 2.75 & 4.69 \\
4 & 5.31 & 4.16 & 6.82 \\
5 & 6.00 & 5.37 & 7.93 \\
6 & 6.61 & 5.99 & 8.05 \\
7 & 6.87 & 6.52 & 8.52 \\
8 & 6.91 & 6.82 & 8.94 \\
9 & 7.60 & 6.91 & 9.28 \\
10 & 8.27 & 7.34 & 9.62 \\
11 & 8.55 & 7.49 & 10.52 \\
12 & 8.67 & 7.61 & 10.70 \\
13 & 10.42 & 8.03 & 12.49 \\
14 & 10.66 & 8.58 & 12.50 \\
15 & 10.86 & 9.15 & 13.98 \\
16 & 11.63 & 9.29 & 14.22 \\
17 & 12.87 & 9.97 & 14.36 \\
18 & 12.92 & 10.71 & 15.86 \\
19 & 13.09 & 11.01 & 16.19 \\
20 & 13.35 & 11.84 & 16.19 \\
21 & 13.71 & 12.15 & 16.32 \\
22 & 14.79 & 13.11 & 16.55 \\
23 & 14.96 & 13.26 & 16.61 \\
24 & 15.06 & 13.77 & 16.62 \\
25 & 15.12 & 14.38 & 16.94 \\
\hline
\end{tabular}




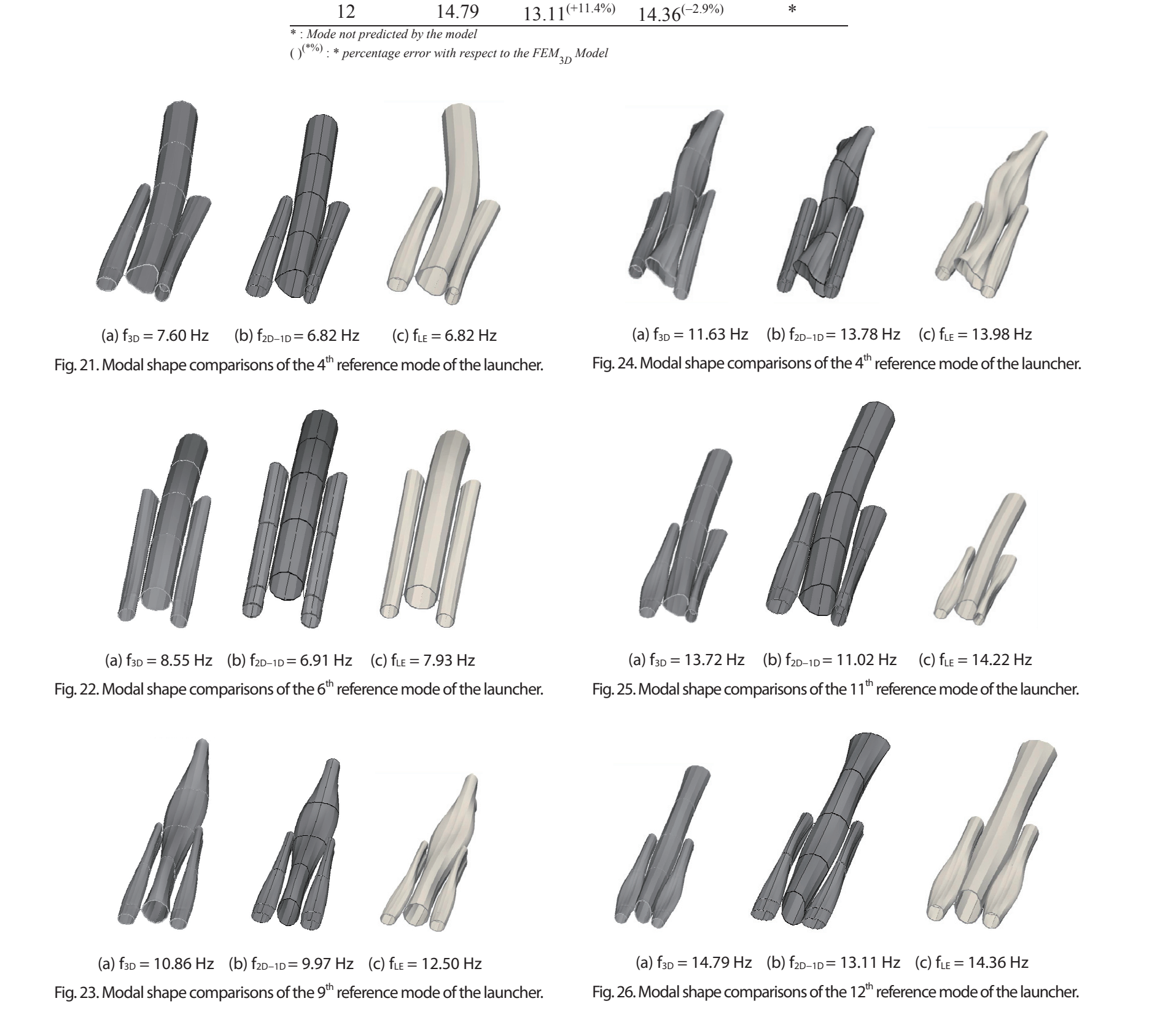


structures reinforced using longitudinal and transversal stiffeners. Two different one-dimensional refined models were derived by means of the Carrera Unified Formulation: one based on the Taylor expansion and the other on the Lagrange expansion. Three reinforced thin-walled structures were taken into account during analysis, including a stiffened plate, a reinforced cylinder and a complete schema of the launcher. The results were compared to those obtained by means of Solid and shell/beam FE models built using the MSC NASTRAN commercial code. Following the analysis, the following conclusing can be drawn:

- the refined one-dimensional models overcome the limitations of the classical models and are able to detect both global and local (shell like) modes.

- the present refined 1D models allow thin-walled and stiffener components to be analyzed by referring to the same element as in the 3D case which is not the case in 2D-shell analysis where stiffeners are usually introduced by beam elements with an off-set;

- refined one-dimensional LE models are at least as accurate as the $1 \mathrm{D}-2 \mathrm{D}$ models even though they require a much smaller number of DOFs; and

- the LE models provide a better solution with respect to the TE models for the analysis of thin-walled structures.

In conclusion, the models that are herein proposed are very attractive for the analysis of reinforced structures. An extension to more complex cases, with the inclusion of a dynamic response and composite structures as well, could be a future extension of the present investigation.

\section{References}

[1] Wempner, G. A. and Wilms, E.V., Multi-rail launcher with six degrees of freedom, University of Alabama Research Institute, 1966.

[2] Przemieniecki, J.S., "Matrix structural analysis of substructures", AIAA Journal, Vol. 1, No.1, 1963, pp.138-147.

[3] Hurty,W.C., "Dynamic analysis of structural systems using component modes", AIAA Journal, Vol. 3, No.4, 1965, pp. 678-685.

[4] Craig, R.R. and Bampton, M.C.C., "Coupling of substructures for dynamic analysis" AIAA Journal, Vol.6, No.7, 1968, pp.1313-1319.

[5] Grimes, P.J., McTigue, L.D., Riley, G.F. and Tilden, D.I., Advancements in structural dynamic technology resulting from Saturn V programs, NASA CR-1539, June 1970.

[6] Benfield, W.A. and Hruda, R.F. "Vibration analysis of structures by component mode substitution", AIAA Journal,
Vol. 9, No.7, 1971, pp.1255-1261.

[7] Goldenberg, S. and Shapiro, M., A study of modal coupling procedures for the space shuttle, NASACR-112252, 1973.

[8] Agrawal, B.N., "Mode synthesis technique for dynamic analysis of structures", The Journal of the Acoustical Society of America, Vol.59, 1976, pp.1329-1338.

DOI:10.1121/1.381019.

[9] Thornton, E.A., Vibration analysis of a 1/15 scale dynamic model of a space shuttle configuration, NASA CR111984, 1971.

[10] Urgueira, A.P.V., Dynamic analysis of coupled structures using experimental data, University of London, 1989.

[11] Friswell, M. and Mottershead, J.E., Finite Element Model Updating in Structural Dynamics, Kluwer Academic Publishers, 1996.

[12] Buehrle, R.D., Fleming, G.A. and Pappa, R.S., "Finite element model development and validation for aircraft fuselage structures". 18th International Modal Analysis Conference, San Antonio, Texas, 2000.

[13] Hu, H., Wang, Y. and Lu, W., "Structural dynamic analysis of a sounding rocket during the liftoff", Journal of Aeronautics, Astronautics ans Aviation, Vol.41, No.2, 2009, pp.111-120.

[14] De Vivo, A., Brutti, C. and Leofanti, J.L., "Modal shape identification of large structure exposed towind excitation by operational modal analysis technique", Mechanical Systems and Signal Processing, Vol.39, 2013, pp.195-206.

[15] Fransen, S., Rixen, D., Henricksen, T. and Bonnet, M., "On the operational modal analysis of solid rocket motors", In: Proceedings of the 28th International Modal Analysis Conference, Jacksonville, Florida, USA, February 1-4, 2010.

[16] Işik, S.K., Ider, C. and Acar, B., "Modeling and verification of a missile launcher system", Journal of Multibody Dynamics, Vol. 228, 2014, pp. 100-107.

[17] Carrera, E., "A class of two dimensional theories for multilayered plates analysis". Atti Accademia delle Scienze di Torino, Memorie Scienze Fisiche, Vol. 19-20, 1995, pp. 49-87.

[18] Carrera, E., "Theories and finite elements for multilayered, anisotropic, composite plates and shells", Archives of Computational Methods in Engineering, Vol.9, No. 2, 2002, pp.87-140.

[19] Carrera, E., "Theories and finite elements for multilayered plates and shells: a unified compact formulation with numerical assessment and benchmarking", Archives of Computational Methods in Engineering, Vol.10, No.3, 2003, pp. 216-296.

[20] Carrera, E. and Giunta, G., "Refined beam theories based on a unified formulation", International Journal of Applied Mechanics, Vol. 2, No. 1, 2010, pp.117-143. 
[21] Carrera, E., Giunta, G., Nali, P. and Petrolo, M., "Refined beam elements with arbitrary cross-section geometries", Computers \& Structures, Vol.88. No.5-6, 2010, pp.283-293.

[22] Carrera, E., Petrolo, M. and Zappino, E., "Performance of CUF approach to analyze the structural behavior of slender bodies", Journal of Structural Engineering, Vol.138, No. 2, 2012, pp. 285-297. DOI:10.1061/(ASCE)ST.1943$541 \mathrm{X} .0000402$.

[23] Carrera, E., Petrolo, M. and Nali, P., "Unified formulation applied to free vibrations finite element analysis of beams with arbitrary section", Shock and Vibrations, Vol. 18, No. 3, 2011, pp. 485-502.

[24] Carrera, E., Pagani, A. and Petrolo, M., "Classical, refined and component-wise analysis of reinforced-shell structures", AIAA Journal, Vol. 51, No. 5, 2013, pp. 1255-1268.

[25] Carrera, E., Pagani, A. and Petrolo, M., "Componentwise method applied to vibration of wing structures", Journal of Applied Mechanics, Vol. 88, No. 4, 2013, pp. 041012.1041012.15.

[26] Carrera, E., Pagani, A. and Petrolo, M., "Classical, refined, and component-wise analysis of reinforced-shell wing structures". AIAA Journal, Vol. 51, No. 5, 2013, pp. 1255-1268.

DOI:10.2514/1.J052331.

[27] Carrera, E., Gaetano, G. and Petrolo M., Beam Structures, Classical and Advanced Theories, John Wiley \& Sons, 2011.

[28] Carrera, E., Cinefra, M., Petrolo, M. and Zappino, E., Finite Element Analysis of Structures Through Unified Formulation, John Wiley \& Sons, 2014. 